\title{
Master curves for the sulphur assisted crosslinking reaction of natural rubber in the presence of nano- and nano-structured $\mathrm{sp}^{2}$ carbon allotropes
}

\author{
S. Musto, V. Barbera, V. Cipolletti, A. Citterio, M. Galimberti ${ }^{*}$ \\ Politecnico di Milano, Department of Chemistry, Materials and Chemical Engineering 'G. Natta', Via Mancinelli 7 , \\ 20131 Milano, Italy
}

Received 23 October 2016; accepted in revised form 19 January 2017

\begin{abstract}
In this paper, master curves are reported for the crosslinking of a diene rubber with a sulphur based system in the presence of either nano- or nano-structured carbon allotropes, such as carbon nanotubes (CNT), a nanosized graphite with high surface area (HSAG) and carbon black (CB). Poly(1,4-cis-isoprene) from Hevea Brasiliensis was the diene rubber and crosslinking was performed in temperatures ranging from 151 to $180^{\circ} \mathrm{C}$, with carbon allotropes below and above their percolation threshold. Such carbon allotropes were characterized by different aspect ratio, surface area and $\mathrm{pH}$. However, in the crosslinking reaction, they revealed common behaviour. In fact, the specific interfacial area could be used to correlate crosslinking parameters, such as induction time $\left(t_{\mathrm{s} 1}\right)$ and activation energy $\left(E_{\mathrm{a}}\right)$ calculated by applying the autocatalytic model. Monotonous decrease of $t_{\mathrm{s} 1}$ and increase of $E_{\mathrm{a}}$ were observed, with points lying on master curves, regardless of the nature of the carbon allotropes. Remarkable differences were however observed in the structure of the crosslinking network: when the carbon allotrope was above the percolation threshold much larger crosslinking density was obtained in the presence of CNT whereas composites based on HSAG became soluble in hydrocarbon solvent, after the reaction with a thiol. Proposed explanation of these results is based on the reactivity of carbon allotropes with sulphur and sulphur based compounds, demonstrated through the reaction of 1-dodecanethiol and sulphur with CNT and HSAG and with a model substrate such as anthracene.
\end{abstract}

Keywords: nanocomposites, carbon allotropes, natural rubber, sulphur, crosslinking

\section{Introduction}

Polymer nanocomposites based on nanosized carbon allotropes are a hot research topic in the materials field. Carbon nanotubes (CNT), both single [1, 2] and multi-walled [3, 4], graphene $(G)[5-8]$ or graphitic nanofillers made of a few layers of graphene [9-12] are used to improve properties such as mechanical reinforcement, electrical and thermal conductivity, thermal and flame resistance, of thermoplastic [13-15], elastomeric [14-18] and thermoset [19-21] matrices. Such nanosized carbon allotropes can be dispersed as individual particles in the polymer matrix and, thanks to their high surface area, can establish large interaction with the polymer, much larger than the one observed with nanostructured carbon black (CB), whose primary particles are fused together to form aggregates [22]. Carbon black and carbon nanofillers have been separately investigated for quite a long time. Recently, features such as crystallinity, shape anisotropy, density, surface area and oil absorption of CNT, a nanosized graphite and CB have been comparatively investigated [23, 24]. Moreover, studies on synergistic effects have been performed, both in thermoplastic [15] and elastomeric matrices [24-27]. In particular, in poly(1,4-cis-isoprene) as the polymer matrix, the synergistic effect on the mechanical

\footnotetext{
*Corresponding author, e-mail: maurizio.galimberti@polimi.it (C) BME-PT
} 
reinforcement of nanosized carbon allotropes and CB has been clearly shown $[25,26]$ and correlation has been established between the filler-polymer interfacial area and the initial modulus of composites with $\mathrm{CNT}, \mathrm{CB}$ and with the hybrid filler system: a master curve was obtained, able to fit all the experimental points $[25,26]$.

It is well known that elastomeric composites, in order to find large scale application, have to be not only reinforced but also crosslinked. In most cases, crosslinking is performed with sulphur based systems [28]. This work was aimed at investigating the effect of carbon allotropes on the crosslinking reaction of a diene elastomer promoted by a sulphur based system. Carbon allotropes were selected with high surface area (s.a.) to favour high interfacial area with polymer matrix. They were: CNT, a nano-sized graphite and CB with s.a. higher than $100 \mathrm{~m}^{2} / \mathrm{g}$. Composites were prepared with the most important diene elastomer: poly(1,4-cis-isoprene) from hevea brasiliensis, natural rubber (NR). Crosslinking reaction was investigated by means of rheometric tests performed at different temperatures. Parameters such as induction and optimum time of crosslinking, minimum and maximum modulus were experimentally determined. The kinetics of the vulcanization reaction was studied through different models and eventually the autocatalytic one, which yielded the best fitting of experimental data, was selected to calculate activation energy. Correlation between data arising from the crosslinking reaction and features of carbon allotropes and composites, such as the specific interfacial area, is presented. Moreover, the reaction between CNT, the nanosized graphite with high surface area (HSAG) and sulphur based systems was investigated. In order to elaborate a tentative interpretation of the experimental results, an alternative mechanism for the interaction of the crosslinking system with the carbon allotropes is proposed.

\section{Experimental part}

\subsection{Materials}

All materials were used as received, without any purification.

Poly(1,4-cis-isoprene) from hevea brasiliensis (NR) (EQR-E.Q. Rubber, BR-THAI, Eastern GR. Thailandia - Chonburi) had trade name STR20 and 73 Mooney Units (MU) as Mooney viscosity (ML(1+4) $\left.100^{\circ} \mathrm{C}\right)$.
Multiwalled carbon nanotubes (CNT) were Baytubes C150 P from Bayer Material Science. The following data were reported in the technical data sheet: chemical purity $95 \%$ by weight, length in the $1-10 \mu \mathrm{m}$ range, a number of walls between 3 and 15 and outer and inner diameters of 10-16 nm and $4 \mathrm{~nm}$, respectively. They were used as received, without any treatment. Nanosized graphite (HSAG) was Synthetic Graphite $8427^{\circledR}$ from Asbury Graphite Mills Inc., with $99,5 \%$ as carbon content. Carbon Black N326 and Carbon Black N110 were from Cabot, with 30 and $15 \mathrm{~nm}$ as mean diameter of spherical primary particles, respectively.

For the preparation of the elastomeric composites, ingredients were: $\mathrm{ZnO}$ (Zincol Ossidi) Stearic acid (Sogis), Sulphur (Solfotecnica), $N$-ter-butyl-2-benzothiazyl sulfenamide (TBBS) (Flexyis) 1-dodecanethiol, azobisisobutyronitrile (AIBN), Anthracene were from Aldrich.

\subsection{Reaction of carbon allotropes with sulphur based compounds}

Reactions were performed in a $100 \mathrm{~mL}$ two-neck round bottomed flask, equipped with magnetic stirrer and kept under nitrogen atmosphere.

\section{Reaction of CNT with 1-dodecanethiol}

$7.6 \mathrm{~g}(9 \mathrm{~mL})$ of 1-dodecanethiol were poured into the flask, $0.18 \mathrm{~g}$ of CNT were added and sonication was performed at room temperature for 1 hour, with a bath sonicator. $0.05 \mathrm{~g}$ of AIBN were then added and the reaction mixture was kept at $80^{\circ} \mathrm{C}$ for 5 hours under stirring. The solid was suspended in ethanol, filtered and washed until 1-dodecanethiol was not detectable in the washing ethanol, through GC-MS analysis.

\section{Reaction of HSAG with 1-dodecanethiol}

$0.5 \mathrm{~g}$ of HSAG (6.9 mmol of aromatic rings), $2.36 \mathrm{~mL}$ of 1-dodecanethiol $(9.9 \mathrm{mmol})$ and $0.05 \mathrm{~g}$ of AIBN $(304 \mu \mathrm{mol})$ were poured into the flask. After 20 minutes at room temperature, the mixture was heated at $70^{\circ} \mathrm{C}$ and maintained at this temperature for 3 hours. The resulting mixture was cooled to room temperature and $7 \mathrm{~mL}$ of ethanol were added. The solution was centrifuged at $3000 \mathrm{rpm}$ for 20 minutes. Upon removing the liquid, $7 \mathrm{~mL}$ of ethanol were added and the suspension was again centrifuged at $3000 \mathrm{rpm}$ for 20 minutes. Upon verifying the absence 
of the thiol in washing ethanol, $0.64 \mathrm{~g}$ of solid were finally recovered.

\subsection{Characterization of carbon allotropes $p H$}

$\mathrm{pH}$ of carbon allotropes was determined according to the standard ASTM D1512 method.

\section{Brunauer-Emmett-Teller (BET) surface area}

BET of carbon allotropes was determined according to the ASTM D6556 method. Nitrogen adsorption at liquid nitrogen temperature $\left(-196^{\circ} \mathrm{C}\right)$ was used to measure surface areas of the samples with a Gemini (Micromeritics) instrument. Before measurements, powders were degassed at $300^{\circ} \mathrm{C}$ under nitrogen flow for 30 minutes. The surface area values were determined by using multi - point BET analysis.

\section{Dibutyl Phthalate (DBP) absorption}

DBP absorption numbers were determined with ASTM D2414 methods, performing the measurements at room temperature with a Absorptometer $\mathrm{C}$ (Brabender $\mathrm{GMbH} \& \mathrm{Co} . \mathrm{kt}$ ) instrument. Before the adsorption measurement, carbon allotropes were dried at $125^{\circ} \mathrm{C}$ for 1 hours.

\section{Wide-angle X-ray diffraction (WAXD)}

Patterns were taken, on pristine carbon allotropes and on the composites, in reflection, with an automatic Bruker D8 Advance diffractometer, with nickel filtered $\mathrm{Cu}-\mathrm{K} \alpha$ radiation $(1.5418 \AA)$, at $35 \mathrm{kV}$ and $40 \mathrm{~mA}$. The intensities of reflections were not corrected for polarization and Lorentz factors, to allow a better visibility of $(00 \ell)$ peaks. $d$-spacings were calculated using Bragg's law [26]. The $D_{\mathrm{hk} \ell}$ correlation length of CNT and HSAG crystals was determined applying the Scherrer equation (Equation (1) [29]):

$D_{\mathrm{hk} \ell}=\frac{K \lambda}{\beta_{\mathrm{hk} \ell} \cos \theta_{\mathrm{hk} \ell}}$ where: $K$ is the Scherrer constant, $\lambda$ is the wavelength of the irradiating beam (1.5419 $\AA, \mathrm{CuK} \alpha)$, $\beta_{\mathrm{hk} \ell}$ is the width at half height, and $\theta_{\mathrm{hk} \ell}$ is the diffraction angle. The introduction of a correction factor has to be used in case $\beta_{\mathrm{hk} \ell}$ is lower than $1^{\circ}$.

Values from the carbon allotropes characterization are reported in Table 1.

\section{Thermogravimetric analysis (TGA)}

TGA tests under flowing $\mathrm{N}_{2}(60 \mathrm{~mL} / \mathrm{min})$ were performed with a Mettler TGA SDTA/851 instrument according to the standard method ISO9924-1. Samples (10 mg) were heated from 30 to $300^{\circ} \mathrm{C}$ at $10^{\circ} \mathrm{C} / \mathrm{min}$, kept at $300^{\circ} \mathrm{C}$ for $10 \mathrm{~min}$, and then heated up to $550^{\circ} \mathrm{C}$ at $20^{\circ} \mathrm{C} / \mathrm{min}$. After being maintained at $550^{\circ} \mathrm{C}$ for $15 \mathrm{~min}$, they were further heated up to $650^{\circ} \mathrm{C}$ with a heating rate of $30^{\circ} \mathrm{C} / \mathrm{min}$ and kept at $650^{\circ} \mathrm{C}$ for $20 \mathrm{~min}$ under flowing air $(60 \mathrm{~mL} / \mathrm{min})$.

\subsection{Reaction of anthracene with 1-dodecanethiol}

$1 \mathrm{~mL}$ (4 mmol) of 1-dodecanethiol was poured in a two rounded bottom flask and it was maintained under vacuum for $20 \mathrm{~min}$. After that $\mathrm{N}_{2}$ was fluxed in-

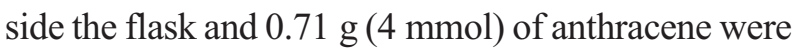
added. After 10 minutes, $0.02 \mathrm{~g}$ of 2-2'-azoisobutyronitrile (AIBN) were added. Then the reaction mixture was heated to $70^{\circ} \mathrm{C}$ for $12 \mathrm{~h}$. The reaction was stopped just by drying, obtaining $0.8 \mathrm{~g}$ of yellow crystals.

\subsection{Reaction of anthracene with sulfur}

Anthracene $(0,100 \mathrm{~g}, 0.6 \mathrm{mmol})$ and sulfur $(12 \mathrm{mmol})$ were poured in a reaction vial equipped with cap and magnetical stirrer. The reaction mixture was maintained under stirring for 12 hours at $151{ }^{\circ} \mathrm{C}$. After this time the mixture was cooled to room temperature, solubilized in chloroform, filtered and analyzed by GC-MS (gas chromatography - mass spectrometry). EIMS $m / z 400[\mathrm{M}]+, 272,176$.

Table 1. $\mathrm{pH}$, BET surface area, DBP absorption number ${ }^{\mathrm{a}}$ and number of graphene layers stacked in the crystalline domain for carbon allotropes (n.d. $=$ not determined)

\begin{tabular}{|l|c|c|c|c|c|}
\hline \multirow{2}{*}{ Carbon allotrope } & $\mathbf{p H}$ & $\begin{array}{c}\text { BET surface area } \\
{\left[\mathbf{m}^{\mathbf{2}} / \mathbf{g}\right]}\end{array}$ & $\begin{array}{c}\text { DBP absorption number } \\
{[\mathbf{m L} / \mathbf{1 0 0} \mathbf{g}]^{\mathbf{a}}}\end{array}$ & \multicolumn{2}{|c|}{ Number of stacked layers } \\
\cline { 3 - 6 } & & 77 & 85 & 113 & 5 \\
\hline CB N326 & $5.7-9.7$ & 137 & 316 & n.d. & 10 \\
\hline CB N110 & $6.9-9.5$ & 200 & 162 & n.d. \\
\hline CNT & n.d. & 330 & 12 & 35 \\
\hline HSAG & 4.6 & & & \\
\hline
\end{tabular}

${ }^{\mathrm{a}} \mathrm{mL}$ of absorbed DBP/100 grams of carbon allotrope 


\subsection{Preparation of composites}

Composites were prepared using a Brabender ${ }^{\circledR}$ type internal mixer, with $50 \mathrm{~mL}$ mixing room.

The standard ASTM D3184 - 89 formulation, shown in Table 2, was adopted as the recipe, as compounds were based on natural rubber. In fact, such formulation is a standard formulation for natural rubber based compounds. Carbon allotropes were fed in two different contents: 4 and $35 \mathrm{phr}$.

The rubber was introduced into the mixer at $80^{\circ} \mathrm{C}$ and masticated for $1 \mathrm{~min}$, with rotors rotating at $60 \mathrm{rpm}$. The filler was added and mixing was carried out for $5 \mathrm{~min}$. The masterbatch was discharged at a temperature ranging from 110 to $115^{\circ} \mathrm{C}$ and fed again to the mixer after $16 \mathrm{~h}$, leaving it in the mixer at $60^{\circ} \mathrm{C}$ and $30 \mathrm{rpm}$ for $1 \mathrm{~min}$. Vulcanization ingredients were added and mixing was performed for further $5 \mathrm{~min}$. All the composites were finally homogeneized by passing them 5 times on a two roll mill operating at room temperature. Such mixing procedure has been already adopted in previous works [24-26] and allowed to obtain even distribution and dispersion of the carbon fillers, both nano- and nano-structured, as revealed by transmission electron microscopy. In particular, composites based on synthetic poly(isoprene) were prepared with amount $30 \mathrm{phr}$ of the same CB and CNT used in the present work and with $33.3 \mathrm{phr}$ of HSAG.

\subsection{Characterization of composites \\ Crosslinking reaction}

It was studied at $151,160,170,180^{\circ} \mathrm{C}$ with a Monsanto oscillating disc rheometer (MDR 2000), determining the torque, expressed in $\mathrm{dNm}$, required for the disc oscillation. Minimum modulus $M_{\mathrm{L}}$, the maximum modulus $M_{\mathrm{H}}$, the modulus $M_{\text {final }}$ at the end of the crosslinking reaction, the time $t_{\mathrm{s} 1}$ required to have a torque equal to $M_{\mathrm{L}}+1$, the time $t_{90}$ required to achieve $90 \%$ of the maximum modulus $M_{\mathrm{H}}$. $t_{90}$ is calculated from $M_{\mathrm{H}}-M_{\mathrm{L}}$. Rheometric experiments were repeated three times for each sample and, in the graph, the average curve is reported. The variation coefficient was found to be below 3\%.

Crosslink density was determined by equilibrium swelling measurements performed in toluene as the
Table 2. Compounds formulation (standard formulation: ASTM D 3184 - 89)

\begin{tabular}{|l|r|r|}
\hline \multicolumn{1}{|c|}{ Ingredients } & phr & phr \\
\hline NR & 100.0 & 100.0 \\
\hline Filler $^{\mathrm{b}}$ & 4.0 & 35.0 \\
\hline ZnO & 6.0 & 6.0 \\
\hline Stearic acids & 0.5 & 0.5 \\
\hline Sulphur & 3.5 & 3.5 \\
\hline TBBS & 0.7 & 0.7 \\
\hline
\end{tabular}

${ }^{\mathrm{b}} \mathrm{CB}$ N326, CB N110, CNT, HSAG

solvent, at $20^{\circ} \mathrm{C}$. Specimens for the measurement were $1 \mathrm{~mm} \times 15 \mathrm{~mm} \times 15 \mathrm{~mm}$.

\section{Total crosslink density}

Before performing the measurement of total crosslink density, samples were swollen in n-heptane for two days under nitrogen atmosphere, to allow reagents' diffusion. N-heptane was then drained and samples were washed with petroleum ether and dried under reduced pressure overnight at room temperature. Samples were then immersed in $200 \mathrm{~mL}$ of toluene, in a glass tube flashed with nitrogen and closed with a cap, and were left in the dark for 72 hours, time commonly considered suitable to reach the equilibrium swelling state. Samples were then taken from the glass tube, the excess of solvent was removed by blotting the samples with a filter paper. Samples were finally and rapidly placed in a clean closed small container and were weighed. Samples were then dried for 24 hours under vacuum at $70^{\circ} \mathrm{C}$, to remove the solvent, and were then weighed again, to obtain the weight of the dry network and the amount of sorbed solvent.

Flory-Rehner equation (Equation (2) [30]), was then used to calculate the cross-link density:

$$
v_{\mathrm{e}}=\frac{-\left[\ln \left(1-V_{\mathrm{r}}\right)+V_{\mathrm{r}}+\chi_{1} V_{\mathrm{r}}^{2}\right]}{\frac{V_{1}\left(V_{\mathrm{r}}^{1 / 3}-V_{\mathrm{r}}\right)}{2}}
$$

where $v_{\mathrm{e}}$ : effective number of chains in a real network per unit volume, $V_{\mathrm{r}}$ : volume fraction of polymer in a swollen network in equilibrium with pure solvent and is calculated as shown in Equation (3):

$$
V_{\mathrm{r}}=\frac{\frac{\text { Weight of dry rubber }}{\text { Density of dry rubber }}}{\frac{\text { Weight of dry rubber }}{\text { Density of dry rubber }}+\frac{\text { Weight of solvent absorbed by sample }}{\text { Density of dry rubber }}}
$$


$\chi_{1}$ : polymer-solvent interaction parameter $(0.391$ at $25^{\circ} \mathrm{C}$ for cis polyisoprene as polymer and toluene as solvent), $V_{1}$ : molecular volume of solvent.

\section{Mono and di-sulfidic crosslinks measurement}

For this measurement, the following procedure was adopted. In a $200 \mathrm{~mL}$ beaker, $100 \mathrm{mg}$ of the crosslinked composite and $100 \mathrm{~mL}$ of heptane were placed, in sequence. The sample was left in heptane for 24 hours without stirring and were then added $4 \mathrm{~mL}$ of piperidine and $3.8 \mathrm{~mL}$ of propanethiol, leaving this mixture at room temperature for 2 hours. The mixture was first washed with heptane (3 times with $50 \mathrm{~mL}$ ) and then filtered. The solid was left again in heptane $(50 \mathrm{~mL})$. After 24 hours, the solvent was removed, the solid was washed with petroleum ether ( 3 times with $50 \mathrm{~mL}$ ) and then filtered using a vacuum pump. The solid obtained was left in $50 \mathrm{~mL}$ of petroleum ether for 2 hours, then was filtered under vacuum. Further drying was performed under vacuum for 24 hours. The resulting sample was again immersed in $200 \mathrm{~mL}$ of toluene for 72 hours, to achieve the equilibrium swelling state. The same procedure reported above for the measurement of the total crosslink density was followed.

\section{Correction to take into account the presence of carbon filler}

It is well known that rubber chains are absorbed on filler particles, which act as physical crosslinks and create swelling restrictions, enhancing the apparent crosslink density. In order to obtain the actual crosslink density, correction was introduced according to ref. [31], estimating the correction factor as a function of DBP absorption number.

\section{Determination of degree of curing $\alpha$}

The degree of curing $\alpha$ was determined through Equation (4).

$\alpha=\frac{M_{\mathrm{t}}-M_{0}}{M_{\mathrm{fin}}-M_{0}}$

where $M_{\mathrm{t}}$ is the modulus (torque) at given curing time $t, M_{0}$ is the modulus at $t=0, M_{\mathrm{fin}}$ is the modulus at the end of the crosslinking reaction.

\section{Determination of kinetics parameters via autocatalytic model}

Kinetic parameters were determined by applying the autocatalityc model, through Equation (5):

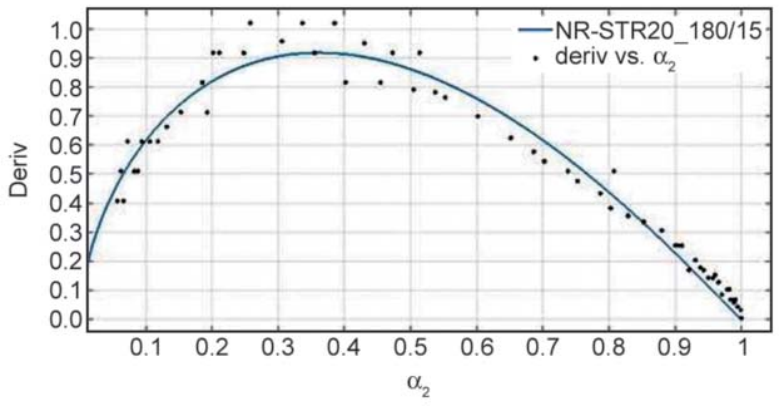

Figure 1. Linear multiple regression analysis of the experimental data

$\frac{\mathrm{d} \alpha}{\mathrm{d} t}=k(T) \alpha^{\mathrm{m}}(1-\alpha)^{\mathrm{n}}$

where $m$ is the autocatalytic index, $n$ is the catalytic index and $k$ is the kinetic constant.

The values of $k, n$, and $m$, at the different temperatures, were calculated with MatLab through linear multiple regression analysis of the experimental data, as reported in Figure 1.

\section{Determination of the activation energy}

The activation energy was calculated with the Arrhenius equation (Equation (6)):

$k=A \cdot \mathrm{e}^{-\mathrm{E}_{\mathrm{a}} / \mathrm{RT}}$

A plot of $\ln K$ versus $1 / T$ gives a straight line of slope $\left(-E_{\mathrm{a}} / R\right)$, where $E_{\mathrm{a}}$ is the activation energy generated during the vulcanization reaction and $R$ is the gas universal constant.

\section{Determination of bound rubber}

Bound rubber was determined on uncrosslinked composite by using toluene as the solvent, by applying the following procedure. $200 \mathrm{mg}$ of each sample were cut into small pieces and placed in a stainless steel wire mesh cage. The cage was immersed in $25 \mathrm{~mL}$ of toluene for nine days at room temperature and the solvent was renewed every three days. At the end of this period, the cage and the composite were dried for one day at room temperature in air and then for 24 hours at $70^{\circ} \mathrm{C}$ under vacuum. Bound rubber was calculated through Equation (7):

$R_{\mathrm{H}}=\frac{W_{\mathrm{fg}}-W \frac{m_{\mathrm{f}}}{m_{\mathrm{f}}+m_{\mathrm{p}}}}{W \frac{m_{\mathrm{p}}}{m_{\mathrm{f}}+m_{\mathrm{p}}}} \cdot 100$

where $W_{\text {fg }}$ is the weight of carbon black and gel, $m_{\mathrm{f}}$ is the weight of the filler in the compound, $m_{\mathrm{p}}$ is the 
weight of the polymer and $W$ is the weight of the specimen.

\section{Crosslinking reaction of composites based on NR and carbon allotropes}

\subsection{Experimental data}

Characteristics of carbon allotropes selected for the present study are shown in Table 1: $\mathrm{pH}$, surface area, absorption of di-isobutyl-phtalate, number of graphene layers stacked in crystalline domains, both in the pristine allotrope and in the crosslinked composite. $\mathrm{pH}$ values lie in the range from about 5 to about 10. It is worth observing that different samples of the same type of carbon black revealed different $\mathrm{pH}$. Surface area was determined through BET measurements. It can be seen that CNT, CB N110 and HSAG have large surface area, higher than $130 \mathrm{~m}^{2} / \mathrm{g}$. In particular, very high surface area was determined for HSAG. Surface area values were used to calculate the specific polymer-filler interfacial area $A_{\mathrm{i}}$, through the product s.a. $* \rho * \phi$, where s.a. is the surface area, $\rho$ is the filler density and $\phi$ is the filler volume fraction. The oil absorption of carbon allotrope was determined in order to investigate the real accessibility of the carbon allotropes to the polymer chains. Correlation between surface area and oil absorption appears for CNT, CB N110 and for the CB grade with lower surface area, CB N326, whereas the oil absorption for HSAG appears relatively low. The number of graphene layers stacked in crystalline domains was calculated by applying the Scherrer equation (see experimental section) to the (002) reflection in the XRD pattern, as already reported [23]. For HSAG, such number is about 35 in its pristine state and increases to about 70 in the crosslinked composite. The number of stacked layers appears to increase also for CNT, from the pristine allotrope to the crosslinked composite. As already commented in previous publications [25,26], a remarkable pressure is applied on the rubber sheet, during the crosslinking step. As a consequence, the rubber composite moves towards a minimum of energy and the graphitic aggregates achieve a higher degree of order.

Formulations adopted for the preparation of the composites are shown in Table 2. Two series of composites were prepared, with two contents of carbon allotropes, 4 and 35 parts per hundred rubber [phr], as the objective was to study the effect of carbon allotropes in the absence and in the presence of continuous filler network. In fact, in previous studies, it was found that the percolation threshold in poly $(1,4-$ cis-isoprene) matrix occurred at about 7,21 and $30 \mathrm{phr}$ for CNT [24], HSAG [25, 26] and CBN326 [25] respectively.

Sulphur-based crosslinking reactions of all of the composites were studied through rheometric analysis.

Table 3 and Table 4 report values of ts 1 and $t_{90}$ measured at $151,160,170$ and $180^{\circ} \mathrm{C}$, for composites with 4 and $35 \mathrm{phr}$ of carbon allotrope, respectively. $t_{\mathrm{s} 1}$ and $t_{90}$ indicate induction and optimum time of crosslinking, respectively.

Table 3. Kinetic parameters from rheometric tests for the crosslinking of composites with $4 \mathrm{phr}$ of different carbon allotropes

\begin{tabular}{|c|c|c|c|c|c|c|c|}
\hline Filler & $\begin{array}{c}\boldsymbol{T} \\
{\left[{ }^{\circ} \mathbf{C}\right]} \\
\end{array}$ & $\begin{array}{c}t_{\mathrm{s}} \\
{[\mathrm{min}]}\end{array}$ & $\begin{array}{c}t_{90} \\
{[\mathrm{~min}]}\end{array}$ & $k$ & $m$ & $n$ & $\begin{array}{c}E_{\mathrm{a}} \\
{[\mathrm{kJ} / \mathrm{mol}]}\end{array}$ \\
\hline \multirow{4}{*}{ 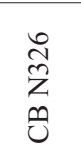 } & 151 & 6.5 & 12.6 & 0.6 & 0.6 & 1.3 & \multirow{4}{*}{85} \\
\hline & 160 & 3.7 & 7.1 & 1.2 & 0.8 & 1.3 & \\
\hline & 170 & 1.8 & 3.6 & 2.1 & 0.8 & 1.2 & \\
\hline & 180 & 1.1 & 1.9 & 3 & 0.7 & 1 & \\
\hline \multirow{4}{*}{$\begin{array}{l}\stackrel{0}{\Xi} \\
\text { Zे } \\
\stackrel{p}{u}\end{array}$} & 151 & 6.6 & 12.6 & 0.4 & 0.3 & 1 & \multirow{4}{*}{114} \\
\hline & 160 & 3.5 & 6.9 & 1 & 0.6 & 1.2 & \\
\hline & 170 & 1.8 & 3.5 & 1.7 & 0.6 & 1.1 & \\
\hline & 180 & 1.1 & 1.9 & 3.1 & 0.7 & 1 & \\
\hline \multirow{4}{*}{ 它 } & 151 & 6.2 & 12.7 & 0.6 & 0.6 & 1.3 & \multirow{4}{*}{92} \\
\hline & 160 & 3.4 & 7 & 1.1 & 0.8 & 1.2 & \\
\hline & 170 & 1.7 & 3.6 & 2.1 & 0.9 & 1.2 & \\
\hline & 180 & 0.9 & 1.9 & 3 & 0.7 & 1 & \\
\hline \multirow{4}{*}{ 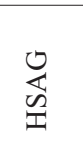 } & 151 & 4.2 & 9.5 & 0.7 & 1 & 1.5 & \multirow{4}{*}{81} \\
\hline & 160 & 2.5 & 5.4 & 2.4 & 1.1 & 1.6 & \\
\hline & 170 & 1.4 & 2.9 & 2.8 & 0.9 & 1.3 & \\
\hline & 180 & 0.9 & 1.7 & 4.3 & 0.9 & 1.2 & \\
\hline
\end{tabular}

Table 4. Kinetic parameters from rheometric tests for the crosslinking of composites with $35 \mathrm{phr}$ of different carbon allotropes

\begin{tabular}{|c|c|c|c|c|c|c|c|}
\hline Filler & $\begin{array}{c}\boldsymbol{T} \\
{\left[{ }^{\circ} \mathrm{C}\right]}\end{array}$ & $\begin{array}{c}t_{\mathrm{s1}} \\
{[\mathrm{min}]}\end{array}$ & $\begin{array}{c}t_{90} \\
{[\mathrm{~min}]}\end{array}$ & $\boldsymbol{k}$ & $m$ & $n$ & $\begin{array}{c}E_{\mathrm{a}} \\
{[\mathrm{kJ} / \mathrm{mol}]}\end{array}$ \\
\hline \multirow{4}{*}{ 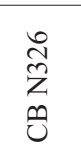 } & 151 & 4.2 & 11.1 & 0.6 & 0.6 & 1.4 & \multirow{4}{*}{91} \\
\hline & 160 & 2.2 & 6.2 & 1 & 0.7 & 1.2 & \\
\hline & 170 & 1.2 & 3.3 & 2 & 0.7 & 1.3 & \\
\hline & 180 & 0.7 & 1.8 & 3 & 0.6 & 1.1 & \\
\hline \multirow{4}{*}{ 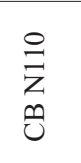 } & 151 & 4.3 & 12.6 & 0.3 & 0.3 & 1.0 & \multirow{4}{*}{100} \\
\hline & 160 & 2.2 & 6.8 & 0.6 & 0.4 & 1.0 & \\
\hline & 170 & 1.2 & 3.5 & 1.2 & 0.5 & 1.0 & \\
\hline & 180 & 0.7 & 1.9 & 2.6 & 0.6 & 1.0 & \\
\hline \multirow{4}{*}{ 艺 } & 151 & 2.3 & 11.3 & 0.4 & 1 & 1.1 & \multirow{4}{*}{118} \\
\hline & 160 & 1.3 & 6.1 & 0.8 & 1.3 & 1.1 & \\
\hline & 170 & 1.2 & 3.3 & 1.4 & 1.2 & 1.1 & \\
\hline & 180 & 0.7 & 1.8 & 2.7 & 1.0 & 1.0 & \\
\hline \multirow{4}{*}{ 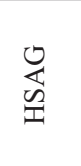 } & 151 & 3.1 & 66.1 & 1.4 & 1.2 & 1.1 & \multirow{4}{*}{154} \\
\hline & 160 & 1.3 & 27.7 & 1.7 & 1.3 & 1.0 & \\
\hline & 170 & 1.2 & 11.5 & 2.3 & 1.4 & 1.1 & \\
\hline & 180 & 0.9 & 5.1 & 3.8 & 1.2 & 1.0 & \\
\hline
\end{tabular}



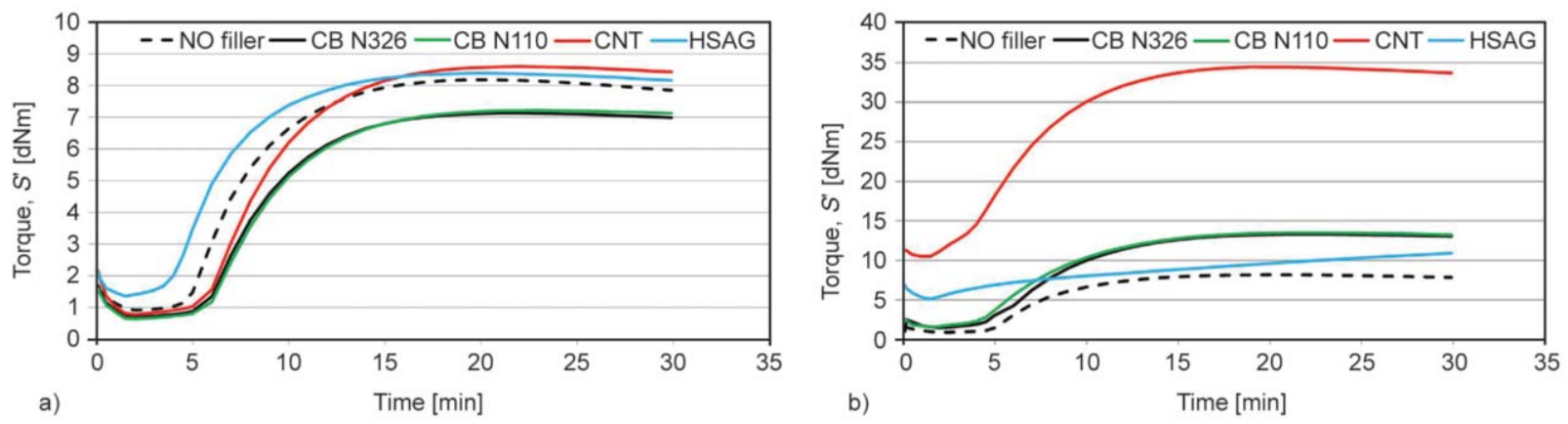

Figure 2. Rheometer curves obtained at $151^{\circ} \mathrm{C}$ for NR based composites containing different carbon allotropes contents: $4 \mathrm{phr}(\mathrm{a}), 35 \mathrm{phr}(\mathrm{b})$

Figure 2 shows the rheometer curves obtained at $151{ }^{\circ} \mathrm{C}$ for composites either without carbon allotropes or containing $4 \mathrm{phr}$ (Figure $2 \mathrm{a}$ ) or $35 \mathrm{phr}$ (Figure b) of one carbon allotrope.

From the inspection of data in Table 3 and in Table 4, the following comments can be made. Crosslinking reaction became faster by increasing the temperature, in the range from 151 to $180^{\circ} \mathrm{C}$ : values of $t_{\mathrm{s} 1}$ and $t_{90}$ consistently decrease. This finding was quite expected. At the lowest temperatures, 151 and $160^{\circ} \mathrm{C}$, lower values of $t_{\mathrm{s} 1}$ were obtained with carbon allotropes having higher surface area, CNT and HSAG. However, the analysis of $t_{90}$ values reveals a peculiar behavior of HSAG: the optimum time of vulcanization is slightly lower at $4 \mathrm{phr}$ and considerably higher at $35 \mathrm{phr}$, at all the crosslinking temperatures. Crosslinking reaction became faster in the presence of carbon allotropes above their percolation threshold (35 phr), as revealed by curves in Figure 2, taken at $151^{\circ} \mathrm{C}$. At $4 \mathrm{phr}$ as carbon allotrope content, crosslinking was accelerated only by HSAG. Curves in Figure 2 show also that the increase of carbon allotrope content leads to the increase of the highest composite modulus $\left(M_{\mathrm{H}}\right)$. CNT based composites achieve the highest $M_{\mathrm{H}}$ values, below and, in particular, above the percolation threshold. The $M_{\mathrm{H}}$ of composites with HSAG increases to a minor extent, passing from 4 to $35 \mathrm{phr}$ as the carbon allotrope content and, at $35 \mathrm{phr}$, becomes lower than $M_{\mathrm{H}}$ of composites based on $\mathrm{CB} . M_{\mathrm{H}}$ values are usually correlated to the structure of the crosslinking network, i.e. with density and length of the sulphur bridges. Crosslinking density was determined through swelling measurements, by applying the Flory-Rehner equation [30], and the length of the sulphur bridges was assessed through the so called thiol-amine chemistry, as described in the experimental part. Data from the analysis of the crosslinking network are shown in
Table 5. Crosslinking density and length of sulphidic bridges for composites based on NR and 4 and $35 \mathrm{phr}$ of carbon allotropes, crosslinked with sulphur based system

\begin{tabular}{|c|c|c|c|c|}
\hline $\begin{array}{l}\text { Carbon } \\
\text { allotrope }\end{array}$ & phr & $\begin{array}{c}\text { Total X-link } \\
{\left[\mathrm{mol} / \mathrm{g} \cdot \mathbf{1 0}^{-5}\right]}\end{array}$ & $\begin{array}{l}\text { Mono and } \\
\text { di-sulfide } \\
\text { [\% mass] }\end{array}$ & $\begin{array}{c}\text { Poly- } \\
\text { sulfidic } \\
{[\% \text { mass }]}\end{array}$ \\
\hline CB N326 & \multirow{4}{*}{4} & 2.6 & 37 & 63 \\
\hline CB N110 & & 2.7 & 32 & 68 \\
\hline CNT & & 3.8 & 27 & 73 \\
\hline HSAG & & 2.9 & 22 & 78 \\
\hline CB N326 & \multirow{4}{*}{35} & 3.2 & 30 & 70 \\
\hline CB N110 & & 2.4 & 27 & 73 \\
\hline $\mathrm{CNT}$ & & 6.5 & 29 & 71 \\
\hline HSAG & & 2.4 & $*$ & $*$ \\
\hline
\end{tabular}

*Not determinable

Table 5. Details for the determination of crosslinking density and of the length of sulphur bridges are in the experimental part.

The highest crosslinking density was found in composites based on CNT, in particular above the percolation threshold of the carbon filler. Composites based on the other carbon allotropes show similar crosslinking density. The distribution of sulphur bridges length is characterized by the largely prevailing amount of polysulphidic bridges. At $4 \mathrm{phr}$ as carbon allotrope content, composites based on CNT and HSAG have lower amount of short sulphur bridges. At $35 \mathrm{phr}$ as carbon allotrope content, similar distribution of bridges was detected in the presence of the two CB grades and CNT, whereas HSAG revealed a peculiar behavior: composites became soluble when the thiol-amine reaction was performed.

Experiments were also carried out in order to determine bound rubber values of binary composites, containing only the rubber and the carbon allotrope. It was not possible to evaluate bound rubber for composites with $4 \mathrm{phr}$ of filler, because samples were dissolved during the analysis. On the contrary, bound 
Table 6. Values of bound rubber for composites based on NR and $35 \mathrm{phr}$ of carbon allotropes

\begin{tabular}{|l|c|}
\hline Carbon allotrope & Bound rubber \\
\hline CB N326 & 22.1 \\
\hline CB N110 & 31.5 \\
\hline CNT & 43.7 \\
\hline HSAG & 46.8 \\
\hline
\end{tabular}

rubber was evaluated for composites based on $35 \mathrm{phr}$ of carbon allotropes. Data are shown in Table 6.

Data in Table 6 show that bound rubber increases with the surface area and is particularly high for nanofillers such as CNT and HSAG.

\subsection{Elaboration of experimental data}

Data from rheometric measurements performed at $151,160,170$ and $180^{\circ} \mathrm{C}$ was elaborated in order to study the kinetics of the crosslinking reactions. The torque measured during the rheometric test, that indicated the stiffness of the composite, was assumed proportional to the crosslinking density. The torque value at $t=0$, at a given curing time $t$ and at the end of the crosslinking reaction were determined and the degree of curing $(\alpha)$ was calculated from Equation (4) reported in the Experimental part. The dependence of $\alpha$ on $t$ and $T$ was investigated by means of three different models [32-36]: induction inflation point [32], exponential [34] and autocatalytic [35, 36].

The latter model is the one discussed here, as it gave the best fitting of experimental data (see Figure 1). In an autocatalytic crosslinking, the maximum rate occurs at a conversion degree other than zero because the reaction is promoted by some of the reaction products. The autocatalytic model is described by Equation (5) reported in the Experimental part and is based on the following parameters: the kinetic constant $(k)$, the catalytic index $(n)$ and the autocatalytic index $(m)$. Values of such kinetic parameters are shown in Table 3 and in Table 4, for 4 and $35 \mathrm{phr}$ as carbon allotrope content, respectively. It appears that, with the increase of crosslinking temperature, $k$ values consistently increase, whereas variation of both catalytic and autocatalytic indexes can not be appreciated. The Arrhenius equation (Equation (6) in the Experimental part) was then adopted to calculate the activation energy $\left(E_{\mathrm{a}}\right)$ of the crosslinking reaction. Values are shown in Table 3 and 4, for composites with carbon allotropes at 4 and $35 \mathrm{phr}$ content, respectively. It is well known that the activation energy is an indication of temperature effect on a reaction: the lowest is $E_{\mathrm{a}}$, the largest is the variation of $k$ with temperature. As shown by data in Table 4, lowest values of $t_{90}$ at the highest temperature are obtained in the presence of low $E_{\mathrm{a}}$ values.

It was then attempted to identify criteria suitable for the rationalization of the data so far reported, aiming in particular at correlating the behaviour of the crosslinking reaction with the features of the carbon allotropes. As mentioned in the introduction, correlation has been established in the literature between the initial modulus of composites based on CNT, CB and on the hybrid CNT/CB system and the filler-polymer interfacial area, deriving a master curve able to fit all the experimental points $[25,26]$. Also in this work, the specific interfacial area, calculated as reported above, was selected as the parameter for correlating data arising from crosslinking reactions.

Graph in Figure 3 reports the values of ts 1 induction time (values are in Table 3 and Table 4), normalized with respect to $t_{\mathrm{s} 1}$ value of the neat matrix, as a function of the specific interfacial area.

It appears that the induction time of the crosslinking reaction almost monotonously decreases as the interfacial area increases, achieving a sort of plateau for interfacial area values close to $60(1 / \mu \mathrm{m})$. A fitting line has been drawn $(R=0.901)$.

Correlation was also attempted between the activation energy (values are in Table 3 and in Table 4) and the specific interfacial area, as it is shown in the graph in Figure 4.

Good correlation can be seen between $E_{\mathrm{a}}$ values and the specific interfacial area. It appears that the activation energy increases with the specific surface area. A fitting line has been drawn $\left(R^{2}=0.9883\right)$. To confirm the existence of a plateau, for low values of surface area, more data would be needed.

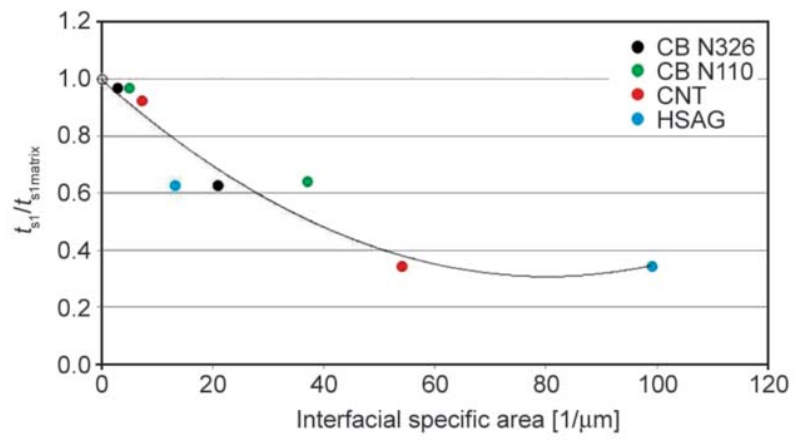

Figure 3. $t_{\mathrm{s} 1} / t_{\mathrm{s} 1}$ matrix ratio as a function of specific interfacial area for NR based composites vulcanized at $151^{\circ} \mathrm{C}$ 


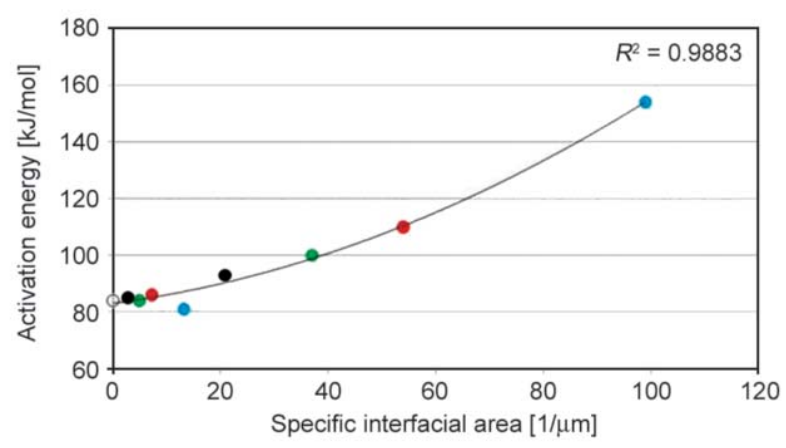

Figure 4. Trend of activation energy with interfacial specific area for compounds based on NR

\subsection{Discussion}

The good correlation, observed in graphs of Figure 3 and Figure 4, between specific interfacial area and either the induction time or the activation energy of crosslinking, indicates that carbon allotropes quite different from each other, such as CNT, a nanosized graphite and two CB grades, have the same behaviour in sulphur based crosslinking of natural rubber. This intriguing result is unprecedented and deserves indeed to be discussed.

The correlation with the specific interfacial area is based on the assumption that the surface area determined through BET measurements is the actual surface of carbon allotropes accessible to the other composite ingredients. As a matter of fact, actual accessibility of carbon allotropes to the polymer chains is better indicated by the oil absorption. As mentioned above in the text, good correlation between surface area and oil absorption was found for CB N326, CB N110 and CNT, whereas the oil absorption of HSAG appeared relatively low. This result could be justified if we take into consideration that graphene layers in HSAG are stacked in crystalline domains, more accessible to the small nitrogen molecules used for BET analysis than to the polymer chains. It is worth noting that the number of graphene layers stacked in a crystalline domain was found to increase (from 35 to 70, see Table 1) from pristine HSAG to the crosslinked composite. This indicates a further reduction of the actual HSAG/polymer interfacial area. The increase of the number of stacked layers, observed also for CNT (see Table 1), could be due to the energy applied during vulcanization, which brings the carbon allotropes to their minimum level of energy, i.e. to the most crystalline aggregate. It is also worth reporting that the master curve, able to correlate composite initial modulus with interfacial areas $[25,26]$, could not fit points of HSAG based composites. In line with what was discussed before, this was explained with the lower actual accessibility of HSAG to the polymer chains. In conclusion, values of interfacial area for HSAG based composites in Figure 3 and Figure 4 could be overestimated. This means that the reduction of induction time and the increase of activation energy could be more pronounced.

Graph in Figure 2 shows that the crosslinking reaction (at $151^{\circ} \mathrm{C}$ ) becomes faster as the interfacial area with the carbon allotrope increases. It is widely acknowledged that carbon allotropes promote faster vulcanization reactions. This was reported for $\mathrm{CB}$, showing that degree of cross-linking and cure rate increased and optimum cure time and scorch time decreased by increasing surface area and sulphur content [3739]. A lot of data is available for carbon allotropes in isoprene matrix, both NR [40-43] and synthetic isoprene rubber $[25,26]$. In the case of CNT, single walled CNT were shown to reduce the activation energy and to accelerate vulcanization [40]. Acceleration of curing was found also in the presence of $\mathrm{CB} / \mathrm{CNT}$ hybrid system $[25,26,43,44]$. It is worth observing that consistent reduction of scorch and curing time was obtained by increasing the relative amount of CNT in the CB/CNT mixture [42]. In the case of nanosized-graphite, scorch and optimum time of vulcanization were reduced by adding HSAG, more clearly in the absence but also in the presence of $\mathrm{CB}[25,26]$ and by adding expanded graphite to E-SBR, also in the presence of CB [45]. In published papers explanations of these results are not so clearcut. In most cases, they are justified with the larger heat transfer and the basic $\mathrm{pH}$ of the carbon allotropes, though also the residual amount of catalyst used for CNT synthesis is taken into consideration [41]. Correlation with the interfacial area has never been reported. Data shown in Table 1 allows to comment the hypothesis about the basic $\mathrm{pH}$ : the correlation shown in the graph of Figure 2 is based on carbon allotropes whose $\mathrm{pH}$ is in the range from about 5 to about 10 . Hence, the basic $\mathrm{pH}$ can not be taken as the prevailing explanation.

However, the most surprising result of the present work is the one shown in Figure 4: the activation energy of vulcanization increases with the specific interfacial area. As mentioned above, the interfacial area calculated for HSAG could be overestimated and points due HSAG in the graph of Figure 3 could be shifted leftward. This would confirm the plateau region at low interfacial area values and would suggest 
an exponential growth of the activation energy as a function of the interfacial area. A first explanation of such behaviour could make reference to the absorption of the vulcanization chemicals on the carbon allotrope surface.

There are however other unexpected results that could hardly be justified by the considerations so far reported. CNT based composites revealed the largest crosslinking density, with a striking increase when the filler content increased from 4 to $35 \mathrm{phr}$. Composites based on HSAG have the lowest value of $M_{\mathrm{H}}$ at $35 \mathrm{phr}$ (see Figure 2b) among the filled composites, with very minor $M_{\mathrm{H}}$ increase with respect to the composite with $4 \mathrm{phr}$ as HSAG content. Moreover, composites with 35 phr as HSAG content become soluble when undergoing thiol-amine reaction. In order to give a reasonable explanation of these results, the reactivity of carbon allotropes with sulphur based chemicals was investigated.

\section{Reactivity of sulphur with carbon allotropes and hypothesis for the crosslinking}

\subsection{Experimental data}

Reaction of CNT and HSAG with 1-dodecanethiol was performed in the presence of a radical initiator, AIBN, and in the absence of solvents, as described in the experimental part. Elemental analysis of reaction products showed the presence of sulphur bound to both carbon allotropes, 12 and 10 as mass $\%$, for HSAG and CNT respectively. These results were confirmed by TGA analysis, which revealed thermal stability for both adducts up to about $230^{\circ} \mathrm{C}$. Free radical assisted reaction of a thiol with CNT has been recently reported in published papers [46]. Reaction was also performed between 1-dodecanethiol and a polycyclic aromatic compound such as anthracene, selected as model compound of the carbon allotropes, in particular of flat graphene layers (details are in the experimental part). GC-MS analysis of pristine anthracene and of the reaction product confirmed the addition of thiol to the aromatic substrate. Figure 5 shows the ${ }^{1} \mathrm{H}$ NMR spectra of pristine anthracene (Figure 5a) and of the reaction product (Figure 5b). The upfield shift of the peaks due to $\mathrm{H}_{1}, \mathrm{H}_{2}, \mathrm{H}_{3}$ and $\mathrm{H}_{4}$ atoms are due the formation of $\mathrm{C}-\mathrm{S}$ bonds.

Such findings suggest that sulphur addition occurs on the edges of HSAG graphene layers. Indeed,

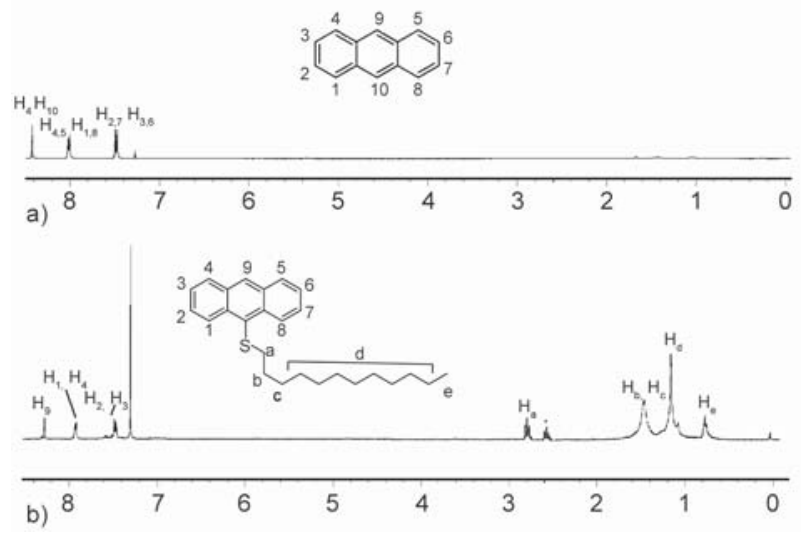

Figure 5. ${ }^{1} \mathrm{H}$ NMR spectra of Anthracene (a) and of the anthracen-9-yl(dodecyl)sulfane reaction product (b)

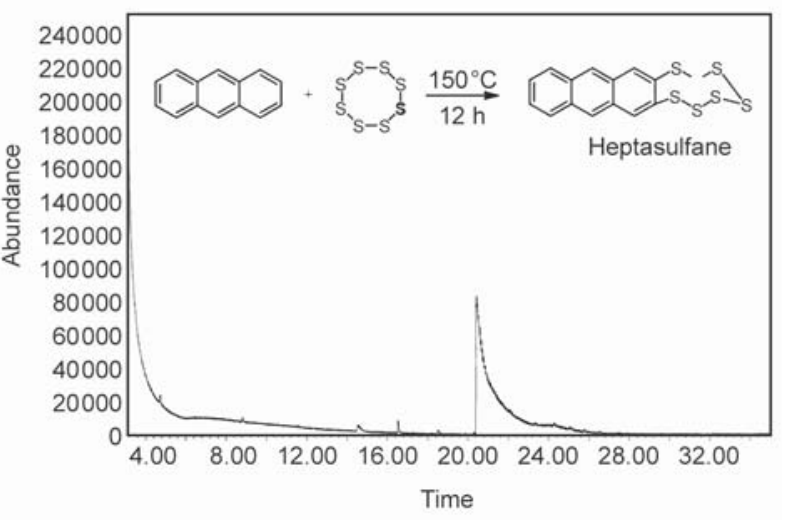

Figure 6. GC-MS spectrum of product of the reaction between anthracene and elemental sulfur

HSAG is characterized by the relevant presence of electronically perturbed edges [47], which could be suitable sites for the reaction with sulphur.

Reaction was also performed between anthracene and sulphur, at $150^{\circ} \mathrm{C}$ for 12 hours (details are in the experimental part). The product of this reaction was analyzed through mass spectrometry, which revealed the formation of only one clearly detectable product. Figure 6 shows the GC-MS spectrum and the identified product: anthraceneheptasulfane. Benzopolysulfanes are naturally occurring products, which contain mainly 3 and 5 sulphur atoms rings. In published paper [48], it is reported that the addition of $\mathrm{S}_{8}$ to 1,2-benzodithiol, in $\mathrm{CH}_{2} \mathrm{Cl}_{2}$, leads to an equilibrium mixture of olysulfanes, with sulphur rings containing 3,5 and 7 atoms.

These findings demonstrate that carbon allotropes with high surface area such as CNT and HSAG are able to react with sulphur and sulphur based compounds. It is worth observing that, in the case of HSAG, functionalization occurs on the edges. 


\subsection{Discussion}

Results reported in the previous paragraph allow to elaborate an alternative working hypothesis for the mechanism of sulphur based crosslinking of unsaturated polymer chain, in the presence of carbon allotropes. The proposed mechanism, sketched in Figure 7, hypothesizes that the carbon allotrope takes part in the vulcanization reaction. In fact, the carbon allotrope is supposedly able to accept electrons from sulphur, with the subsequent formation of radical cations, favouring the quick start of the vulcanization reaction. Electrons, that could remain on carbon, would then be responsible for the higher activation energy.

Such mechanism is helpful for the explanation of data and elaborations reported above.

The largest crosslinking density measured for the CNT based composites could be attributed to the ability of the tubes to take part in the crosslinking network, as single tubes or as entangled coils. This hypothesis could account for the striking increase of crosslinking density of CNT based composites, when the filler content increased from 4 to $35 \mathrm{phr}$. It is worth recalling that CNT have high surface area and that the bound rubber, determined for the composite based on $35 \mathrm{phr}$ of CNT, was large indeed, almost twice as large as the CBN326 based composite. The large CNT-NR interfacial area could definitely favour a pronounced interaction and thus a high crosslinking density. However, it is worth recalling that the value of crosslink density was calculated taking into consideration the presence of the filler, estimating its ability to interact with the polymer matrix on the basis of DBP absorption number (see the experimental part). It is worth adding that, in the frame of the present investigation, other surface features should be considered, such as surface activity and degree of disorder. At present, comparative investigation has not been performed.

Some of the authors reported Raman spectra of HSAG [47]. In a Raman spectrum, lines D and G, located at 1350 and $1590 \mathrm{~cm}^{-1}$ respectively, indicate the presence of graphitic $\mathrm{sp}^{2}$-phase. The $\mathrm{G}$ peak is typical of bulk crystalline graphite (graphene) and the D peak occurs with the presence of either disorder or confinement (e.g. by edges) of the graphitic layers [4954]. HSAG, obtained by milling, revealed a $D$ peak with remarkable intensity. Higher degree of disorder could be expected to bring to larger reactivity. In spite of large surface area and bound rubber and the relatively large degree of disorder, composite with 35 phr of HSAG revealed lower crosslinking density and $M_{\mathrm{H}}$ values with respect to composites with $4 \mathrm{phr}$ and became soluble during the thiol-amine reaction. These findings could be interpreted in the light of the observed ring formation on anthracene substrate. Such ring formation suggests that a polycyclic aromatic compound, such as the graphene layers in HSAG, can seize sulphur. This could prevent the formation of the crosslinking network, thus justifying the decrease of crosslinking density of HSAG based composites when the filler increased from 4 to $35 \mathrm{phr}$, the low value of $M_{\mathrm{H}}$ and the solubility (after of the thiol-amine reaction) of composites with $35 \mathrm{phr}$ of HSAG. The reduction of crosslinking density of N110 based composites, when the filler increased from 4 to $35 \mathrm{phr}$, could be explained in the same way, at least as a tentative working hypothesis.

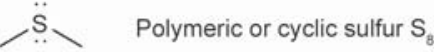

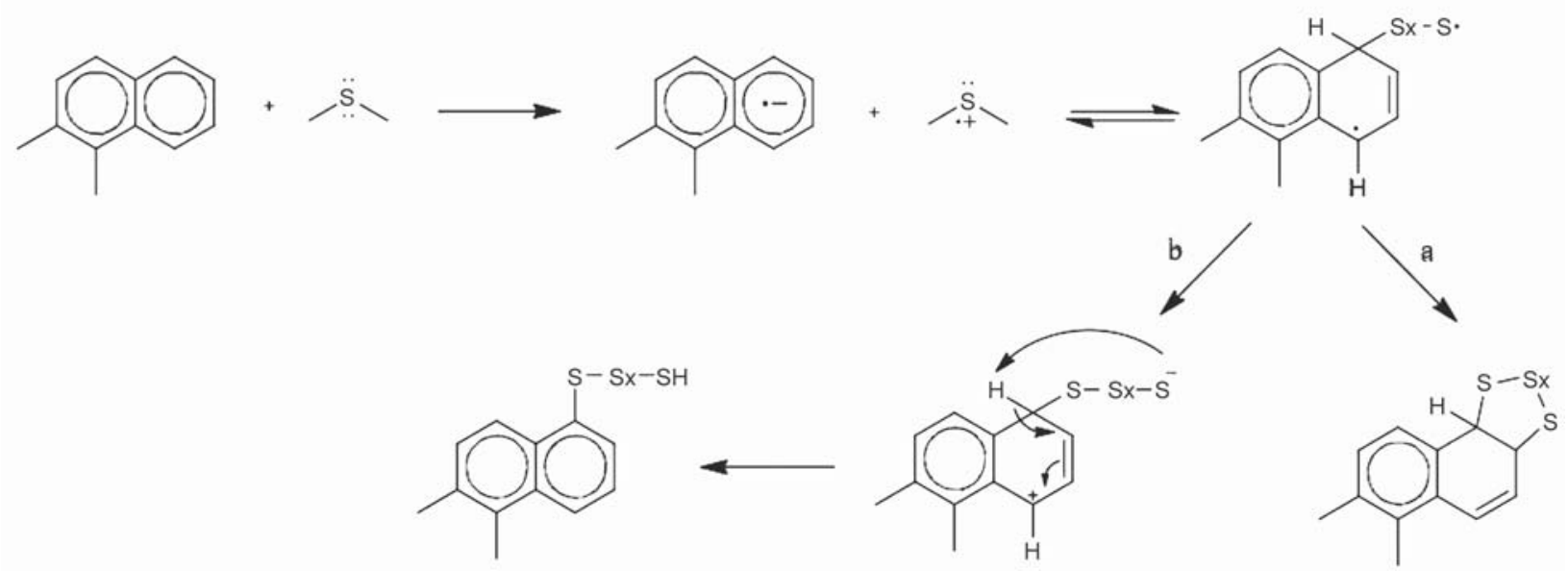

Figure 7. Proposed mechanism for the reaction of sulphur with a carbon allotrope 


\section{Conclusions}

This work demonstrates that carbon allotropes quite different from each other such as CNT, HSAG and different grades of CB have a common behaviour in the sulphur based crosslinking of a diene rubber such as NR. Reasonable explanation of the experimental results could be based on the reactivity of the carbon allotropes with sulphur and with the sulphur based chemicals of the crosslinking system.

Crosslinking reactions were performed in temperatures ranging from 151 to $180^{\circ} \mathrm{C}$, determining induction time $t_{\mathrm{s} 1}$, optimum time $t_{90}$ and activation energy $E_{\mathrm{a}}$ of the reactions, through the application of the autocatalytic model of vulcanization. The interfacial area was found able to correlate $t_{\mathrm{s} 1}$ and $E_{\mathrm{a}}$ values: consistent decrease of $t_{\mathrm{s} 1}$ (normalized with respect to $t_{\mathrm{s} 1}$ of the matrix) up to a plateau region and almost exponential increase of $E_{\mathrm{a}}$, after a short initial plateau, were observed, as a function of the interfacial area. Sort of master curves could be identified. It is well known that the term 'master curve' should be used to describe physical changes in large range of time. However, as reported in the text above, it has been used [22] for describing the correlation between fillerpolymer interfacial area and modulus data of composites with CNT, CB and with hybrid filler systems. In the light of that, master curve is used also in the present work and allows to emphasize the key role played by the interfacial area.

The structure of the crosslinked materials was observed to depend on the carbon allotrope, when its content was above the percolation threshold. Crosslinking density was similar for CB and HSAG and appreciably higher for CNT based composites. Moreover, HSAG based composites were soluble in hydrocarbon solvents, after the reaction with a thiol.

Free radical assisted reactivity of CNT and HSAG with a sulphur compound was demonstrated, preparing adducts of such carbon allotropes with 1-dodecanethiol and performing the reaction of the thiol with anthracene.

Faster vulcanization reaction in the presence of carbon allotropes is prevailingly explained in published papers with better heat transfer and with the basic $\mathrm{pH}$ of the carbon allotropes. The present work advises to critically revise this hypothesis, as $\mathrm{pH}$ values of the carbon allotropes were in a pretty wide range, from acidic to basic values. Larger activation energy could be simply due to the absorption of vulcanization chemicals on the filler surface. An alternative hypothesis, able to justify the common behavior of CNT, HSAG and CB, is proposed here. The quick start of vulcanization could be due to electron transfer from sulphur to the carbon allotrope. Such electrons, lying on the carbon surface could then be responsible for higher activation energy.

\section{Acknowledgements}

Pirelli Tyre is gratefully acknowledged for the financial support, Mrs Anna Gastaldi for her help with the English language.

\section{References}

[1] Iijima S., Ichihashi T.: Single-shell carbon nanotubes of $1 \mathrm{~nm}$ diameter. Nature, 363, 603-605 (1993). https://doi.org/10.1038/363603a0

[2] Bethune D. S., Kiang C. H., de Vries M. S., Gorman G., Savoy R., Vazquez J., Beyers R.: Cobalt-catalysed growth of carbon nanotubes with single-atomic-layer walls. Nature, 363, 605-607 (1993). https://doi.org/10.1038/363605a0

[3] Iijima S.: Helical microtubules of graphitic carbon. Nature, 354, 56-58 (1991). https://doi.org/10.1038/354056a0

[4] Monthioux M., Kuznetsov V. L.: Who should be given the credit for the discovery of carbon nanotubes? Carbon, 44, 1621-1623 (2006). https://doi.org/10.1016/j.carbon.2006.03.019

[5] Novoselov K. S., Geim A. K., Morozov S. V., Jiang D., Zhang Y., Dubonos S. V., Grigorieva I. V., Firsov A. A.: Electric field effect in atomically thin carbon films. Science, 306, 666-669 (2004).

https://doi.org/10.1126/science.1102896

[6] Geim A. K., Novoselov K. S.: The rise of graphene. Nature Materials, 6, 183-191 (2007).

https://doi.org/10.1038/nmat1849

[7] Allen M. J., Tung V. C., Kaner R. B.: Honeycomb carbon: A review of graphene. Chemical Review, 110, 132-145 (2010). https://doi.org/10.1021/cr900070d

[8] Zhu Y., Murali S., Cai W., Li X., Suk J. W., Potts J. R., Ruoff R. S.: Graphene and graphene oxide: Synthesis, properties, and applications. Advanced Material, 22, 3906-3924 (2010). https://doi.org/10.1002/adma.201001068

[9] Geng Y., Wang S. J., Kim J-K.: Preparation of graphite nanoplatelets and graphene sheets. Journal of Colloid and Interface Science, 336, 592-598 (2009). https://doi.org/10.1016/j.jcis.2009.04.005

[10] Kavan L., Yum J. H., Grätzel M.: Optically transparent cathode for dye-sensitized solar cells based on graphene nanoplatelets. ACS Nano, 5, 165-172 (2011). https://doi.org/10.1021/nn102353h 
[11] Nieto A., Lahiri D., Agarwal A.: Synthesis and properties of bulk graphene nanoplatelets consolidated by spark plasma sintering. Carbon, 50, 4068-4070 (2012). https://doi.org/10.1016/j.carbon.2012.04.054

[12] Paton K. R., Varrla E., Backes C., Smith R. J., Khan U., O’Neill A., Boland C., Lotya M., Istrate O. M., King P., Higgins T., Barwich S., May P., Puczkarski P., Ahmed I., Moebius M., Pettersson H., Long E., Coelho J., O’Brien S. E., McGuire E. K., Mendoza Sanchez B., Duesberg G. S., McEvoy N., Pennycook T. J., Downing C., Crossley A., Nicolosi V., Coleman J. N.: Scalable production of large quantities of defect-free few-layer graphene by shear exfoliation in liquids. Nature Materials, 13, 624-630 (2014).

https://doi.org/10.1038/nmat3944

[13] Mutiso R. M., Winey K. I.: Electrical properties of polymer nanocomposites containing rod-like nanofillers. Progress in Polymer Science, 40, 63-84 (2015). https://doi.org/10.1016/j.progpolymsci.2014.06.002

[14] Mittal G., Dhand V., Rhee K. Y., Park S-J., Lee W. R.: A review on carbon nanotubes and graphene as fillers in reinforced polymer nanocomposites. Journal of Industrial and Engineering Chemistry, 21, 11-25 (2015). https://doi.org/10.1016/j.jiec.2014.03.022

[15] Szeluga U., Kumanek B., Trzebicka B.: Synergy in hybrid polymer/nanocarbon composites. A review. Composites Part A: Applied Science and Manufacturing, 73, 204-231 (2015).

https://doi.org/10.1016/j.compositesa.2015.02.021

[16] Bokobza L.: Multiwall carbon nanotube elastomeric composites: A review. Polymer, 48, 4907-4920 (2007). https://doi.org/10.1016/j.polymer.2007.06.046

[17] Galimberti M., Cipolletti V., Kumar V.: Nanofillers in natural rubber. in 'Natural rubber materials: Composites and nanocomposites' (eds.: Thomas S., Chan C. H., Pothan L. A., Ramanan J. M.) Royal Society of Chemistry, London, 34-72 (2014).

https://doi.org/10.1039/9781849737654-00034

[18] Papageorgiou D. G., Kinloch I. A., Young R. J.: Graphene/elastomer nanocomposites. Carbon, 95, 460 484 (2015).

https://doi.org/10.1016/j.carbon.2015.08.055

[19] Prolongo S. G., Jiménez-Suárez A., Del Rosario G., Ureña A.: High mobility of carbon nanotubes into thermosetting matrix. European Polymer Journal, 74, 209 217 (2016).

https://doi.org/10.1016/j.eurpolymj.2015.11.029

[20] Lionetto F., Calò E., Di Benedetto F., Pisignano D., Maffezzoli A.: A methodology to orient carbon nanotubes in a thermosetting matrix. Composites Science and Technology, 96, 47-55 (2014).

https://doi.org/10.1016/j.compscitech.2014.02.016

[21] McClory C., McNally T., Brennan G. P., Erskine J.: Thermosetting polyurethane multiwalled carbon nanotube composites. Journal of Applied Polymer Science, 105, 1003-1011 (2007).

https://doi.org/10.1002/app.26144
[22] Donnet J-B., Custodero E.: Reinforcement of elastomers by particulate fillers. in 'Science and technology of rubber' (eds.: Mark J. E., Erman B., Eirich F. R.) Elsevier, Amsterdam, 367-400 (2005).

https://doi.org/10.1016/B978-012464786-2/50011-0

[23] Mauro M., Cipolletti V., Galimberti M., Longo P., Guerra G.: Chemically reduced graphite oxide with improved shape anisotropy. Journal of Physical Chemistry C, 116, 24809-24813 (2012).

https://doi.org/10.1021/jp307112k

[24] Galimberti M., Coombs M., Riccio P., Riccó T., Passera S., Pandini S., Conzatti L., Ravasio A., Tritto I.: The role of CNTs in promoting hybrid filler networking and synergism with carbon black in the mechanical behavior of filled polyisoprene. Macromolecular Materials and Engineering, 298, 241-251 (2012).

https://doi.org/10.1002/mame.201200075

[25] Agnelli S., Cipolletti V., Musto S., Coombs M., Conzatti L., Pandini S., Riccò T., Galimberti M.: Interactive effects between carbon allotrope fillers on the mechanical reinforcement of polyisoprene based nanocomposites. Express Polymer Letters, 8, 436-449 (2014). https://doi.org/10.3144/expresspolymlett.2014.47

[26] Galimberti M., Kumar V., Coombs M., Cipolletti V., Agnelli S., Pandini S., Conzatti L.: Filler networking of a nanographite with a high shape anisotropy and synergism with carbon black in poly(1,4-cis-isoprene)based nanocomposites. Rubber Chemistry and Technology, 87, 197-218 (2014).

https://doi.org/10.5254/rct.13.87903

[27] Dong B., Liu C., Lu Y., Wu Y.: Synergistic effects of carbon nanotubes and carbon black on the fracture and fatigue resistance of natural rubber composites. Journal of Applied Polymer Science, 132, 42075-42083 (2015). https://doi.org/10.1002/app.42075

[28] Coran A. Y.: Vulcanization. in 'Science and technology of rubber' (Erman B., Mark J. E., Roland C. M.) Elsevier Amsterdam, 337-382 (2005).

[29] Alexander L. E.: X-ray diffraction methods in polymer science. Wiley, New York (1979).

[30] Flory P. J., Rehner Jr J.: Statistical mechanics of crosslinked polymer networks I. Rubberlike elasticity. Journal of Chemical Physics, 11, 512-520 (1943).

https://doi.org/10.1063/1.1723791

[31] Saville B., Watson A. A.: Structural characterization of sulfur-vulcanized rubber networks. Rubber Chemistry and Technology, 40, 100-148 (1967).

https://doi.org/10.5254/1.3539039

[32] Arrillaga A., Zaldua A. M., Atxurra R. M., Farid A. S.: Techniques used for determining cure kinetics of rubber compounds. European Polymer Journal, 43, 4783-4799 (2007).

https://doi.org/10.1016/j.eurpolymj.2007.08.024

[33] Yeoh O. H.: Mathematical modeling of vulcanization characteristics. Rubber Chemistry and Technology, 85, 482-492 (2012).

https://doi.org/10.5254/rct.12.87982 
[34] Armand J. Y., Vergnaud J.: Modelling of processes in calorimetry by taking into account heat transfer and reaction. Thermochimica Acta, 121, 381-395 (1997). https://doi.org/10.1016/0040-6031(87)80188-0

[35] Piloyan G. O., Ryabchikov I. D., Novikova O. S.: Determination of activation energies of chemical reactions by differential thermal analysis. Nature, 212, 1229 (1966). https://doi.org/10.1038/2121229a0

[36] Berglund L. A., Kenny J. M.: Processing science for high performance thermoset composites. SAMPE Journal, 27, 27-37 (1991).

[37] Semsarzadeh M.A., Bakhshandeh G. R., GhasemzadehBarvarz M.: Effect of carbon black on rate constant and activation energy of vulcanization in EPDM/BR and EPDM/NR blends. Iranian Polymer Journal, 14, 573 578 (2005).

[38] Li Z. H., Zhang J., Chen S. J.: Effects of carbon blacks with various structures on vulcanization and reinforcement of filled ethylene-propylene-diene rubber. Express Polymer Letters, 2, 695-704 (2008).

https://doi.org/10.3144/expresspolymlett.2008.83

[39] Thomas S., Stephen R.: Rubber nanocomposites: Preparation, properties, and applications. Wiley, New York (2010).

https://doi.org/10.1002/9780470823477

[40] López-Manchado M. A., Biagiotti J., Valentini L., Kenny J. M.: Dynamic mechanical and Raman spectroscopy studies on interaction between single-walled carbon nanotubes and natural rubber. Journal of Applied Polymer Science, 92, 3394-3400 (2004).

https://doi.org/10.1002/app.20358

[41] Cataldo F., Ursini O., Angelini G.: MWCNTs elastomer nanocomposite, Part 1: The addition of MWCNTs to a natural rubber-based carbon black-filled rubber compound. Fullerenes, Nanotubes and Carbon Nanostructures, 17, 38-54 (2009).

https://doi.org/10.1080/15363830802515907

[42] Ismail H., Ramly A. F., Othman N.: The effect of carbon black/multiwall carbon nanotube hybrid fillers on the properties of natural rubber nanocomposites. Polymer-Plastics Technology and Engineering, 50, 660-666 (2011).

https://doi.org/10.1080/03602559.2010.551380

[43] Thaptong P., Sirisinha C., Thepsuwan U., Sae-Oui P.: Properties of natural rubber reinforced by carbon blackbased hybrid fillers. Polymer-Plastics Technology and Engineering, 53, 818-823 (2014).

https://doi.org/10.1080/03602559.2014.886047

[44] Junkong P., Kueseng P., Wirasate S., Huynh C., Rattanasom N.: Cut growth and abrasion behaviour, and morphology of natural rubber filled with MWCNT and MWCNT/carbon black. Polymer Testing, 41, 172-183 (2015).

https://doi.org/10.1016/j.polymertesting.2014.11.009
[45] Malas A., Pal P., Giri S., Mandal A., Das K. C.: Synthesis and characterizations of modified expanded graphite/emulsion styrene butadiene rubber nanocomposites: Mechanical, dynamic mechanical and morphological properties. Composites Part B: Engineering, 58, 267-274 (2014).

https://doi.org/10.1016/j.compositesb.2013.10.028

[46] Yang X. H., Guo J. W., Yang S., Hou Y., Zhang B., Yang H. G.: A free radical assisted strategy for preparing ultra-small Pt decorated CNTs as a highly efficient counter electrode for dye-sensitized solar cells. Journal of Materials Chemistry A, 2, 614-619 (2014).

https://doi.org/10.1039/C3TA13986G

[47] Galimberti M., Barbera V., Guerra S., Conzatti L., Castiglioni C., Brambilla L., Serafini A.: Biobased Janus molecule for the facile preparation of water solutions of few layer graphene sheets. RSC Advances, 5, 8114281152 (2015).

https://doi.org/10.1039/C5RA11387C

[48] Aebisher D., Brzostowska E. M., Sawwan N., Ovalle R., Greer A.: Implications for the existence of a heptasulfur linkage in natural o-benzopolysulfanes. The Journal of Natural Products, 70, 1492-1494 (2007). https://doi.org/10.1021/np070108t

[49] Castiglioni C., Tommasini M., Zerbi G.: Raman spectroscopy of polyconjugated molecules and materials: Confinement effect in one and two dimensions. Philosophical Transactions of the Royal Society A: Mathematical, Physical and Engineering Sciences, 362, 24252459 (2004).

https://doi.org/10.1098/rsta.2004.1448

[50] Tommasini M., Di Donato E., Castiglioni C., Zerbi G., Severin N., Böhme T., Rabe J. P.: Resonant Raman spectroscopy of nanostructured carbon based materials: The molecular approach. AIP Conference Proceedings, 723, 334-338 (2004).

[51] Graf D., Molitor F., Ensslin K., Stampfer C., Jungen A., Hierold C., Wirtz L.: Spatially resolved raman spectroscopy of single- and few-layer graphene. Nano Letters, 7, 238-242 (2007).

https://doi.org/10.1021/n1061702a

[52] Casiraghi C., Hartschuh A., Qian H., Piscanec S., Georgi C., Fasoli A., Novoselov K. S., Basko D. M., Ferrari A. C.: Raman spectroscopy of graphene edges. Nano Letters, 9, 1433-1441 (2009). https://doi.org/10.1021/n18032697

[53] Radovic L. R., Bockrath B.: On the chemical nature of graphene edges: Origin of stability and potential for magnetism in carbon materials. Journal of the American Chemical Society, 127, 5917-5927 (2005). https://doi.org/10.1021/ja050124h

[54] Casiraghi C., Pisana S., Novoselov K. S., Geim A. K., Ferrari A. C.: Raman fingerprint of charged impurities in graphene. Applied Physics Letters, 91, 233108/123108/3 (2007). https://doi.org/10.1063/1.2818692 